\title{
Pregnancy with HIV positive, esophageal varices and restless leg syndrome
}

\begin{abstract}
Background: India is the third largest HIV epidemic in the world. The prevalence rate of HIV in India was 0.3 in 2013, where 2.1 million people living with HIV and 68,000 deaths were found due to HIV. According to UNICEF Seventh stock taking report of 2016 estimated that in India nearly 35,000 pregnant mothers living with HIV.
\end{abstract}

Objectives: To describe the condition of antenatal mother with retroviral positive, esophageal varices and Restless leg syndrome.

Methods: Detailed history, physical examination and laboratory investigation and treatment.

Conclusion: The concern of health team toward pregnant mother with retroviral positive is more since need of prevention, control and treatment for both mother and the baby. Along with if mother affected with esophageal varices and restless leg syndrome, the condition has to be diagnosed and managed carefully with both medical and surgical treatment as early as possible for the prognosis of mother and child.

Keywords: primigravida, HIV positive, esophageal varices, restless leg syndrome, spleenomegaly
Volume 2 Issue I - 2017

\author{
SP Bhavya \\ Department of Nursing, JSS College of Nursing, India
}

Correspondence: SP Bhavya, Department of Nursing, JSS college of Nursing, India, Email spbhavya 123@gmail.com

Received: December 13, 2016 | Published: January 18, 2017

\section{Introduction}

HIV (Human Immunodeficiency Virus) is a Lentivirus a member of the retrovirus family. The immune system of an HIV-infected person becomes so weakened that it cannot protect itself from serious infections. When this happens, the person clinically has AIDS. ${ }^{1}$

\section{Case report}

An Antenatal mother, Mrs.XXX of 26 years, Primigravida with 36 weeks of gestation got admitted to the hospital with the complaints of back pain since 2days. Her obstetrical history as follows;

\section{I trimester}

She conceived spontaneously after 3 months of her marriage, she confirmed her pregnancy through ultrasonography at 6 weeks of gestation, her first trimester was uneventful.

\section{II trimester}

At $13^{\text {th }}$ week, she diagnosed as retroviral positive, after counselling she was on antiretroviral therapy which include flaviridine $600 \mathrm{mg}$, lavuvidine $300 \mathrm{mg}$, trenforin $300 \mathrm{mg}$ and she continued iron, folic acid and calcium supplements and also she was taken 2 doses of TT injection, on anomaly scan no gross anomaly found. At 22 weeks she had complaints of epigastric pain and abdominal pain on sleep, undergone EVI(Esophageal varices Imaging) for esophageal Varices the result showed large esophageal varices with Restless Leg Syndrome (RSL), for which Esophageal Varices Band Ligation was performed. ${ }^{2}$

\section{III trimester}

On repeat EVI, found small esophageal varices with RLSEVL along with gastric varices portal hypertensive gastropathy. Her height is $557 \mathrm{~cm}$ and weight $47 \mathrm{kgs}$ her vitals were stable, Hb- 9.0mg/dl, RBC- 3.40million/cmm, Total Leukocyte count-3430mill/ cmm, Neutrophil-68.3\%, Esonophile-1.7\%, Monocytes-3.8\%, CBR(memory)-83mg/dl. On Ultrasound abdomen, altered echo texture of liver, spleenomegaly with dilated and tortous portal vein and peripheral texture of portal hypertension.

\section{Human immuno deficiency virology}

In man, the target host cells are CD4 antigen bearing T-helper lymphocytes.

\section{$\downarrow$}

Virus infects these key immune cells and introduces its RNA into the cell.

\section{$\downarrow$}

By attaching to the CD4 receptors and other cell membrane molecule.

\section{$\downarrow$}

The viral enzyme reverse transcriptase uses this RNA as a template for transcription of RNA into DNA,

\section{$\downarrow$}

Which is then incorporated into the host's genome

\section{$\downarrow$}

Subsequently transcribed to produce viral RNA

\section{$\downarrow$}

The genetic material of next generation of viral particles mature,

The mature viral particles from the host cell can infect other cells. The rapid and continuous replication of HIV impairs and eventually 
depletes the CD4 and T cells in patient's body. This progressive debilitation of the immune system and its network renders the patient susceptible to opportunistic infections and characterise AIDS.

\section{Effect of pregnancy on disease}

Plasma HIV viral load and CD4 cell count are the laboratory markers for severity of HIV infection. Its range in healthy adult is 500-1500cells/cum. the absolute CD4 count decreases to $543+169$ cells/cum during pregnancy irrespective of whether they are HIV positive or negative. Pregnancy is not associated with worsening of the disease. ${ }^{3}$

\section{Effect of disease on pregnancy}

Increased incidence of IUGR and preterm delivery has been observed in HIV positive women with low CD4 count and advanced disease. No increased risk of congenital malformations has been observed.

\section{Antenatal care in women with HIV}

All pregnant women who are HIV positive should be screened and treated for genital infections during pregnancy. This should be done as early as possible in pregnancy and repeated around 28 weeks.

\section{Advantages of HIV screening in pregnancy}

i. Patient can choose the option MTP.

ii. Optimal care can be planned if continuation of pregnancy.

iii. Strategies can be implemented to reduce risk of mother to child transmission

iv. Future planning can be done by couples.

v. Plasma viral load and CD4 T-lymphocyte measurements to be reviewed by the HIV physicians at four to six months interval during pregnancy. PCP (pneumocystis carinii pneumonia) prophylaxis is usually administered when the CD4 T-lymphocyte count is below $200 \times 10^{6} / 1$ in the form of Tab Cotrimaxazole (Sulfamethoxazole $800 \mathrm{mg}$ and Trimethoprim $160 \mathrm{mg}$ ) once daily.

vi. Women taking antiretroviral drugs should be monitored for drug toxicity (full blood count, urea and electrolytes, liver function tests, lactate and blood glucose). Presentation with signs and symptoms of pre-eclampsia, cholestasis or other signs of liver dysfunction during pregnancy may indicate drug toxicity and early liaison with HIV physician should be sought.

vii. Fetal anomaly scan is important especially after first trimester in those who have exposure to HAART and folate antagonists used for prophylaxis against PCP.

viii. Intensive fetal monitoring, including assessment of fetal anatomy with a level II ultrasound and continued assessment of fetal growth and well being during the third trimester should be considered for mothers receiving combination of antiretroviral therapy.

\section{Esophageal varices}

Esophageal varices are abnormal, enlarged, tortuous veins in the tube that connects the throat and stomach (esophagus). This condition occurs most often in people with serious liver diseases. Esophageal varices develop when normal blood flow to the liver is blocked by a clot or scar tissue in the liver. To go around the blockages, blood flows into smaller blood vessels that aren't designed to carry large volumes of blood. The vessels can leak blood or even rupture, causing lifethreatening bleeding. ${ }^{4}$

\section{Pathophysiology}

Varicosities occur secondarily to portal hypertension and are a dilation of various alternative pathways when the portal return of blood is obstructed by cirrhosis of liver. Varicosities are most common in the distal third of the esophagus but may occur throughout the esophagus. Varicose of the esophagus is a less common cause of upper GI hemorrhage, but the consequences of bleeding are an everimpending danger to the life of the patient. Bleeding occurs when the tension in the wall of the veins leads to rupture, it may result in shock. Stop spontaneously, the coagulation factors and thrombocytopenia which interferes with hemostasis. ${ }^{5}$

\section{Clinical picture}

Cardinal symptoms of esophageal varicosities are recurrent hematemesis and melena. Patients with acute variceal bleeding have hemodynamic instability $(61 \%)$, tachycardia $(22 \%)$, hypotension (29\%), and orthostatic hypotension (10\%).

\section{Assessment and diagnostic test}

a. Endoscopy, barium swallow ultrasonography, CT and Angiography -to identify the cause and the site of bleeding

b. Portal hypertension measurement-Indirect measurementrequires insertion of a catheter with a balloon into the antecubital or femoral vein.

c. Direct measurement-laparotomy-a needle may be introduced into the spleen a monometer reading of more than $20 \mathrm{ml}$ saline is abnormal.

d. Laboratory test- liver function test, spleenoportography, Hepatoportography, celiac angiography.

\section{Treatment and management}

Variceal management encompasses three phases

i. Prevention of initial bleeding.

ii. Management of acute bleeding.

iii. Prevention of rebleeding.

a. Treatment includes pharmacologic, endoscopic and radiologic shunting and surgery. Once large varices are identified, patients should start with beta-blocker therapy such as propranolol. It reduces portal pressure and variceal blood flow and decreases the risk of bleeding by $50 \%$. Adding isosorbide mononitrate further reduces recurrent bleeding. Hepatic venous pressure measurements are the optimal modality to monitor the success of pharmacological therapy. The combination of pharmacologic therapy has been shown to be superior to sclerotherapy and may be superior to band ligation.

b. Vasopressin is a posterior pituitary hormone that constricts splanchnic arterioles and reduces portal flow and pressure and Prophylactic intravenous antibiotics should also be started. Endoscopy is performed to diagnose and treat hemorrhage. 
c. Definitive therapy is first performed with sclerotherapy or band ligation, successful in $90 \%$ of patients. Varices are injected with sclerosing solutions to stop acute bleeding/ repeated injections will cause variceal obliteration and may prevent recurrent bleeding.

d. When bleeding is under control, endoscopic ligation and sclerotherapy are repeated every 1 to 2 weeks until the varices are eradicated. This technique has the fewest complications and the lowest incidence of recurrence.

e. Surviellance is performed at 3 to 6 month intervals to detect and treat any recurrence. Patients who have two or more rebleeds should be considered for surgery or transplantation.

f. Balloon tamponade is used as bridge to definitive therapy in $6 \%$ of patients when hemostasis is not achieved. Connected balloons in the stomach and the esophagus compress the varices.

g. If medical and endoscopic therapies fail, transjugular intrahepatic portosystemic shunt (TIPS) is the procedure of choice of emergency bleeding TIPS should be reserved for patients who have poor liver function.

h. Patients must be followed up closely because the shunt may occlude in up to $50 \%$ of them within 18 months.

i. Surgical shunts should be used to prevent rebleeding in patients who do not tolerate, or who are noncompliant with, medical therapy and who have relatively preserved liver function.

\section{Course and prognosis}

Overall short-term mortality rates after acute bleeding are $10 \%$ to $15 \%$. However, in patients with cirrhosis who have variceal bleeding, the risk for death is as high as $60 \%$ at 1 year.

\section{Restless leg syndrome}

It is a part of nervous system disorder that causes an urge to move legs, which increases during evening and night while sleeping, lying and sitting. Diagnosis based solely on clinical criteria.

\section{Following are the four essential criteria to diagnose RLS}

a) Urge to move the legs that are usually accompanied by uncomfortable or unpleasant sensations in the legs.

b) Unpleasant sensations or the urge to move will begin or worsen during periods of rest or inactivity such as lying or sitting.

c) Unpleasant sensations or urge to move are partly or totally relieved by movement such as walking, bending, stretching, etcetera, at least as long as the activity continues.

d) Unpleasant sensations worsen in the evening or at night than during the day.

\section{Treatment}
a. Prevent or relieve symptoms.
b. Increase the amount and quality of sleep.

c. Treat or correct any underlying condition.

d. Lifestyle changes can prevent or relieve the symptoms of RLS, for mild RLS, lifestyle changes may be the only treatment needed.

e. Avoiding common substances such as alcohol and tobacco can limit or prevent symptoms.

f. Pharmacological treatment: Dopamine agonists

g. Dopaminergic agents.

h. Benzodiazepines.

i. Opiates.

j. Anticonvulsants.

k. Alpha2 agonists.

1. RLS can become a chronic condition that requires treatment in moderate to severe cases.

m. The available therapies for RLS are pharmacologic and non pharmacological therapies.

The available evidence for the various treatments for RLS with regard to

a) Disease symptoms

b) Quality of life and sleep outcomes

c) Adverse effects

\section{Conclusion}

HIV in pregnancy should be managed by multidisciplinary team, where antenatal care is similar to that of HIV positive women. Here the pregnancy is not considered as high risk simply by virtue of HIV infection but a women with HIV positive with esophageal varices and restless leg syndrome should be considered and treat as high risk pregnancy.

\section{Acknowledgements}

None

\section{Conflict of interest}

The author declares no conflict of interest.

\section{References}

1. Kaizad R Damania, Parikshit D Tank. Federation of obstetric and gynaecological societies of India. Manual of Perinatal Infections. India: Jaypee Publications; p. 51-52.

2. Global HIV Statistics; 2016.

3. Sorbi D, Gostout CJ, Peura D, et al. An assessment of management of acute bleeding varices, a multi center prospective member-based study. AM J Gastroentral. 2003;98(11):2424-2434.

4. Treatments for Restless Legs Syndrome; 2015.

5. Floch Neil R Netter's .Gastroenterology. International student edition. New Jersey, USA: Icon Learning Systems LLC; p. 66-68. 